\title{
Care of patients with a diagnosis of chronic obstructive pulmonary disease: a cluster randomised controlled trial
}

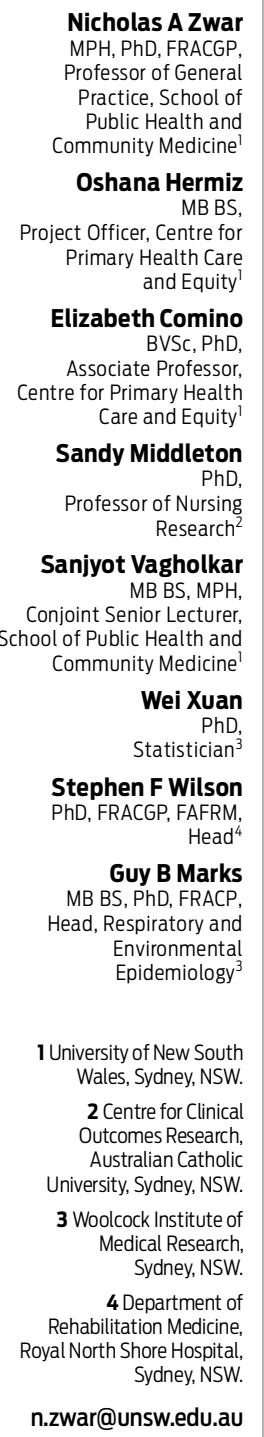

MJA 2012; 197: 394-398 doi: $10.5694 / \mathrm{mjal} 2.10813$

Editorial p 363 hronic obstructive pulmonary disease (COPD) is a leading cause of death worldwide. ${ }^{1}$ Guidelines for care of COPD provide recommendations for slowing disease progression and optimising function in people with COPD. ${ }^{2,3}$ The key interventions are smoking cessation, pulmonary rehabilitation, influenza vaccination, optimising medicines, patient education and effective management of exacerbations.

There is a need for effective approaches to implementation of evidence-based treatment in primary care, where many patients with COPD are managed. Nurses, either within the practice or visiting to provide specialised care, could have a role in improving management of COPD, including by helping to implement planned care.

A review of nine randomised trials of nurse-led chronic disease management for COPD concluded that there was no evidence of improvements in patients' healthrelated quality of life, psychological wellbeing, disability or pulmonary function. ${ }^{4} \mathrm{~A}$ more recent Cochrane review of nine trials of outreach programs involving nurse home visits to COPD patients concluded that providing support and education, monitoring health status and providing liaison with physicians resulted in improved disease-specific quality-of-life measures but had variable effects on hospitalisation. ${ }^{5}$ A New Zealand study, which was not included in the Cochrane Review as it did not have a substantial home-visit component, resulted in reduced hospital admissions and bed-days, and significant improvements in quality of life and lung function. ${ }^{6}$

A previous randomised trial conducted by members of our group evaluated the effect of a brief nurseled intervention, including development of a care plan, after discharge

Objective: To evaluate a partnership model of care for patients with a diagnosis of chronic obstructive pulmonary disease (COPD).

Design, setting and participants: Cluster randomised controlled trial with blinded outcome assessment of 44 general practices in south-western Sydney comprising 451 people with a diagnosis of COPD, conducted between 2006 and 2009.

Intervention: Participants from intervention group practices were visited at their home by a registered nurse with specific training in COPD care who worked with the general practitioner, the patient and other health professionals to develop and implement an individualised care plan based on best-practice guidelines. Participants from control group practices received usual care.

Main outcome measures: The primary outcome was disease-related quality of life measured using the St George's Respiratory Questionnaire (SGRQ) at 12-month follow-up. Other outcomes were overall quality of life, lung function, smoking status, immunisation status, patient knowledge of COPD, and health service use.

Results: Of the 451 participants, 257 (57.8\%) were confirmed as having COPD on post-bronchodilator spirometry. Follow-up was completed for 330 patients (73.2\%). At 12 months, there was no statistically significant difference in the mean SGRQ scores between intervention and control groups (38.7 $\vee 37.6$; difference, 1.1; $95 \% \mathrm{Cl},-1.53-3.74 ; P=0.41$ ) or in measures of quality of life, lung function and smoking status. Compared with the control group, in the intervention group, attendance at pulmonary rehabilitation was more frequent (31.1\% v 9.6\%; OR, 5.16; $95 \% \mathrm{Cl}, 2.40-11.10 ; P=0.002)$ and the mean COPD knowledge score was higher (10.5 v 9.8; difference, 0.70; $\mathrm{Cl}, 0.10-1.21 ; P=0.02$ ).

Conclusion: The nurse-GP partnership intervention did not have an impact on disease-related quality of life at 12-month follow-up. However, there was evidence of improved quality of care, in particular, in attendance at pulmonary rehabilitation and patient knowledge of COPD.

Trial registration: Australian Clinical Trials Registry ACTRN012606000304538.

from hospital on clinical outcomes in patients with COPD. ${ }^{7}$ There was no difference between groups in health-related quality of life or hospital admissions. Patients in the intervention group had higher knowledge scores and were more satisfied with their care. In that study, less than a third of the general practitioners remembered receiving the care plan, and there were no differences in GP visits or management.

We hypothesised that more active engagement of the GPs, and the nurse having a role not only in developing but also implementing a plan of care, would be more effective. Therefore, this study evaluated the effectiveness, compared with usual care, of a nurse with training in COPD care working in partnership with the patient and their GP to develop and implement an individualised care plan based on clinical practice guidelines.

\section{Methods}

\section{Research design}

A cluster randomised trial, with randomisation at the level of the practice, was conducted to avoid contamination between intervention and control groups. The study protocol has been published. ${ }^{8}$ Recruitment started in December 2006 and follow-up was completed in May 2009. Ethics approval was from University of New South Wales and Sydney South West Area Health Service human research ethics committees.

\section{Recruitment}

GPs were recruited from a list of 256 GPs from practices in south-western Sydney who had previously taken part 
in research or who attended continuing medical education activities held by local Divisions of General Practice. GPs on the list were approached by mail, followed by a telephone call from one of the researchers ( $\mathrm{NZ}$ or $\mathrm{SV}$ ). Inclusion criteria for GPs were using an electronic prescribing system and having seen COPD patients in the past 12 months.

Participating GPs, who were not aware of their allocation status at the time of patient recruitment, were asked to search their electronic records to identify patients who had been prescribed medications used for COPD defined as inhaled $\beta_{2}$ agonists, inhaled corticosteroids, ipratropium bromide, tiotropium, oral theophylline and oral corticosteroids. Patients were eligible if they were aged between 40 and 80 years, had been prescribed one or more of these medications, and had seen the GP in the previous 12 months. GPs were then asked to manually review the list generated and identify patients that they considered to have a diagnosis of COPD, emphysema or chronic bronchitis, including those that they considered to have coexisting problems, such as asthma. GPs were asked to include patients regardless of how or where the diagnosis had been made, and information on the diagnostic process was not collected. Patients were excluded if they did not speak English or had significant cognitive impairment.

Letters were sent from the practice to eligible patients inviting them to take part in the study. Two fortnightly reminders were sent to the nonresponding patients.

\section{Randomisation}

A researcher who took no further part in the study randomised practices to intervention or control groups, with allocation concealment. Details of the randomisation process have been published previously. ${ }^{8}$

\section{Intervention}

Two nurses, specifically recruited and trained for this study, worked in partnership with GPs to implement the intervention. In the service model, the nurses were external to the practice and visited patients in their homes. ${ }^{6}$ The training program for the nurses involved attendance at a 2-day work- shop where the following topics were presented by expert clinicians: pathophysiology of COPD; assessment of COPD; spirometry; smoking cessation; management of COPD according to Australian and New Zealand guidelines; ${ }^{3}$ the role of pulmonary rehabilitation in the management of COPD; and the management of exacerbations. The training covered the principles and practice of motivational interviewing and self-management support. Following the training, there were monthly meetings lasting 1-2 hours between the nurses and members of the study team (NZ and S V), and feedback from a respiratory physician on the quality of their spirometry (GM). The intervention and its implementation are described online at mja.com.au. The intervention was delivered between 2007 and 2009.

\section{Control}

GPs in the control-group practices were provided with a copy of the COPD guidelines, and their patients received usual care, which was defined as processes normally followed by the GP and the patient regarding review, pharmacological therapy and management of COPD.

\section{Outcome measures}

The primary outcome measure was disease-related quality of life, measured using the St George's Respiratory Questionnaire (SGRQ) at 12 months after recruitment. The SGRQ is a validated instrument designed to measure the impact of respiratory diseases (in particular, asthma and COPD) on an individual's life. ${ }^{9}$ The SGRQ is scored from zero to 100 , where zero indicates best quality of life and 100, the worst. A change in score of $\geqslant 4$ is considered to be clinically significant. ${ }^{9,10}$

Other outcome measures were overall quality of life (measured using the 12-item Short Form Health Survey [SF-12], which is a generic measure of health impairment); lung function; smoking status; immunisation status for influenza and pneumococcus; attendance at pulmonary rehabilitation; patient knowledge of COPD; and health service use. For those patients with COPD on spirometry, classification of severity was made using Global Initiative for
Chronic Obstructive Lung Disease (GOLD) criteria. ${ }^{2}$ The patient's knowledge of COPD was measured by a 12-item scale developed for a previous project. ${ }^{7}$ This scale asked patients if they knew the name of their chest condition, which immunisations were helpful in reducing the risk of exacerbations, patient actions that could help control symptoms and improve quality of life, and symptoms suggestive of an exacerbation. Correct answers scored one point and incorrect answers zero points, resulting in a score out of 12 . The scale has face validity but has not been subjected to validation testing.

Outcomes, with the exception of the SGRQ, were measured at three points in time: recruitment (baseline), 6 months and 12 months after randomisation. The SGRQ was measured at baseline and 12 months. Assessments were conducted at the participant's residence or at the GP's practice (according to patient preference) by project officers ( $\mathrm{OH}$ and $\mathrm{IH})$ who took no part in the intervention and were blind to group allocation.

\section{Analysis}

The analysis was based on intention to treat. The effect of the intervention on continuous variables was estimated and tested in SAS, version 9.2 (SAS Institute Inc) using a mixedmodel procedure, in which time and treatment groups were fixed-effectand subject-nested within practice clusters, and time points within subjects were random effects. For the binary outcome variables, a generalised estimating equation method was separately implemented at each time point that incorporated practice cluster effect. The analyses for continuous and binary variables were adjusted for baseline values.

Given that the measurement of outcome variables (with the exception of SGRQ score) was done at baseline, 6 and 12 months, an analysis was done to examine whether a time and intervention group interaction was present. This was found not to be the case, so baseline and 12-month measures are reported.

\section{Sample size}

The sample size calculation was based on detecting a between-group 


\section{Baseline characteristics of groups}

\begin{tabular}{lcc} 
Characteristic & Intervention $(n=234)$ & Control $(n=217)$ \\
\hline Mean age, years (SD) & $65.8(10.3)$ & $64.4(10.3)$ \\
No. of men (\%) & $110(47.0 \%)$ & $106(48.8 \%)$ \\
No. of current smokers (\%) & $74(31.6 \%)$ & $61(28.1 \%)$ \\
No. with FEV 1 /FVC $<0.7(\%)$ & $146(62.4 \%)$ & $11(51.2 \%)$ \\
GOLD stage: no. of patients (\%) & & \\
Stage 1 & $28(12.0 \%)$ & $20(9.2 \%)$ \\
Stage 2 & $70(29.9 \%)$ & $63(29.0 \%)$ \\
Stage 3 & $38(16.2 \%)$ & $19(8.8 \%)$ \\
Stage 4 & $10(4.3 \%)$ & $9(4.1 \%)$ \\
Mean SGRQ score (SD) & $42.0(18.3)$ & $42.0(19.4)$ \\
Mean COPD knowledge score (SD) & $8.9(1.4)$ & $8.9(1.5)$ \\
No. vaccinated for influenza (\%) & $156(66.7 \%)$ & $155(71.4 \%)$ \\
No. vaccinated for pneumococcus (\%) & $137(58.5 \%)$ & $123(56.7 \%)$ \\
Mean no. of comorbidities & 3.6 & 4.1 \\
Mean SF-12 score (SD) & & $36.6(12.5)$ \\
Physical component & $36.4(11.1)$ & $49.0(11.4)$ \\
Mental component & $49.3(11.7)$ & \\
\hline
\end{tabular}

$\mathrm{FEV}_{1}==$ forced expiratory volume in 1 second. $\mathrm{FVC}=$ forced vital capacity. SGRQ = St George's Respiratory Questionnaire. GOLD = Global Initiative on Obstructive Lung Disease; stages describe severity of disease from mild (Stage 1) through to severe (Stage 4). COPD = chronic obstructive pulmonary disease. SF-12 $=12$-item Short Form Health Survey.

difference in SGRQ score of $\geqslant 4$ at 12 months after intervention. 9,10 After adjusting for clustering, the number per group required to detect this difference with $80 \%$ power at the $5 \%$ significance level was estimated to be 200 per group, based on an intracluster coefficient of 0.01 and a resultant design effect of 1.09 for a cluster size of 10. Details of the sample size calculation have been published. ${ }^{8}$

\section{Results}

We recruited 56 GPs from 44 practices in south-west Sydney. The mean age of the GPs was 52.3 years and $47 \%$ were men. Participating GPs searched their patient records, identifying 1144 patients who were eligible and invited to participate. Of these, 451 (39.4\%) patients were recruited and provided baseline data, $330(73.2 \%)$ of whom completed the 12-month assessment (see the flow diagram online at mja.com.au). Characteristics of patients completing the 12-month assessment compared with those lost to follow-up were: age, $65.3 \mathrm{v} 64.7$ years; men, $47.3 \%$ v $49.6 \%$; spoke English at home, $79.1 \% \mathrm{v} 79.7 \%$; and current smokers, $30.2 \% \quad v \quad 31.1 \%$. There was a lower rate of confirmed COPD than expected. Of the 451 participants, $445(98.7 \%)$ were able to perform baseline spirometry and, of these, $257(57.8 \%)$ were confirmed to have COPD. ${ }^{11}$

Baseline characteristics of participants are shown in Box 1. There was a higher rate of confirmed COPD, lower forced expiratory volume in 1 second and fewer comorbidities in the intervention group, but the groups did not differ on the SGRQ or other characteristics.
There were no statistically significant between-group differences in the primary outcome measure (SGRQ) or in overall quality of life, respiratory function or smoking status at 12month follow-up (Box 2). The intracluster correlation for the SGRQ calculated using the mixed-model procedure was 0.03 . In continuing smokers, there was a trend towards fewer cigarettes smoked per day in the intervention group.

In process-of-care outcomes, more patients in the intervention group reported having attended a pulmonary rehabilitation program. This difference was statistically significant $(P=0.002)$. Data were not collected on whether they completed the program. There was a higher rate of patients in the intervention group reporting being vaccinated for influenza and pneumococcus, but neither difference was statistically significant. There was no difference in the frequency of either GP or hospital or emergency department attendance in the previous 3 months. The COPD knowledge score was statistically significantly higher in the intervention group $(P=0.02)$ (Box 3$)$.

\section{Discussion}

A notable finding was that only $57.8 \%$ of patients identified as having COPD and being eligible for the study were confirmed as having the condition according to spirometric criteria. We have previously published baseline analyses of the accuracy of diagnosis showing that having a spirometer in the practice was not predictive of agreement between the clinical and spirometric diagnoses. ${ }^{11}$ Older patient age was associated with correct diagnosis, while higher numbers of

\section{Disease-related and overall quality of life, respiratory function and smoking status at 12-month follow-up}

\begin{tabular}{lcccc} 
Outcome & $\begin{array}{c}\text { Intervention } \\
(n=234 \text { randomised }) *\end{array}$ & $\begin{array}{c}\text { Control } \\
(n=217 \text { randomised }) *\end{array}$ & Difference $(95 \% \mathrm{Cl})$ & $P$ \\
\hline SGRQ score & $38.7(19.0)$ & $37.6(19.1)$ & $1.1(-1.53-3.74)$ & 0.41 \\
SF-12 score & & & & \\
$\quad$ Physical component & $36.3(11.6)$ & $36.7(13.0)$ & $-0.4(-2.1-1.3)$ & 0.61 \\
$\quad$ Mental component & $51.0(11.2)$ & $51.5(11.3)$ & $-0.5(-2.3-1.2)$ & 0.55 \\
FEV post-bronchodilator, percentage of predicted (SD) & $72.3 \%(24.1 \%)$ & $73.1 \%(24.4 \%)$ & $-0.8(-3.3-1.7)$ & 0.51 \\
No. of current smokers (\%) & $47 / 161(29.2 \%)$ & $37 / 168(22.0 \%)$ & $1.3(0.6-2.5)^{\dagger}$ & 0.52 \\
No. of cigarettes per day (SD) $(n=157)$ & $15(8)$ & $18(11)$ & $-3(-6.0-0.04)$ & 0.05 \\
\hline
\end{tabular}

* Figures are mean (SD) unless stated otherwise stated. $\dagger$ Odds ratio $(95 \% \mathrm{Cl})$. FEV $\mathrm{FE}_{1}$ forced expiratory volume in 1 second. SGRQ = St George's Respiratory Questionnaire. SF-12 = 12-item Short Form Health Survey. 
3 Process-of-care outcomes at 12-month follow-up

\begin{tabular}{|c|c|c|c|c|}
\hline Patient outcome & Intervention* & Control* & Odds ratio $(95 \% \mathrm{Cl})$ & $P$ \\
\hline No. vaccinated for influenza (\%) & $131 / 161(81.4 \%)$ & $129 / 167(77.2 \%)$ & $1.88(0.88-4.02)$ & 0.13 \\
\hline No. vaccinated for pneumococcus (\%) & $117 / 161(72.7 \%)$ & $103 / 167(61.7 \%)$ & $1.64(0.93-2.89)$ & 0.09 \\
\hline No. attended pulmonary rehabilitation program (\%) & $50 / 161(31.1 \%)$ & $16 / 167(9.6 \%)$ & $5.16(2.40-11.10)$ & 0.002 \\
\hline No. visited general practitioner in preceding 3 months (\%) & $148 / 160(92.5 \%)$ & $150 / 169(88.8 \%)$ & $1.59(0.70-3.61)$ & 0.27 \\
\hline $\begin{array}{l}\text { No. used hospital or emergency department service in } \\
\text { preceding } 3 \text { months (\%) }\end{array}$ & $27 / 161(16.8 \%)$ & $24 / 169(14.2 \%)$ & $1.21(0.70-2.10)$ & 0.50 \\
\hline COPD knowledge score (SD) & $10.5(2.0)$ & $9.8(1.9)$ & $0.7(0.1-1.2)^{\dagger}$ & 0.02 \\
\hline
\end{tabular}

$\mathrm{COPD}=$ chronic obstructive pulmonary disease. $*$ Denominators in columns are number of patients with valid data at 12-month follow-up.

$\dagger$ Difference $(95 \% \mathrm{Cl})$.

comorbidities were associated with misdiagnosis.

The study found no between-group difference in the primary outcome measure (SGRQ) and also no between-group differences in SF-12 score, lung function or smoking rates at 12-month follow-up. Differences in lung function would be unlikely to be apparent within 12 months, so longer term follow-up would be needed to show change in this outcome measure. Smoking cessation is a key priority for COPD care, and about $30 \%$ of participants in the study were smokers. However, we did not succeed in getting more patients in the intervention group to quit. Among continuing smokers, there were fewer cigarettes smoked in the intervention versus the control group, but as the mean difference was only three cigarettes per day, this would be unlikely to produce a health benefit.

There was greater attendance at a pulmonary rehabilitation program by patients in the intervention group, indicating improved delivery of evidence-based care. It has been shown that participation in pulmonary rehabilitation leads to improvements in health-related quality of life (measured by the SGRQ) at the end of the program. ${ }^{12}$ The fact that these benefits were not apparent in our study may be due to insufficient numbers of participants attending and/or completing pulmonary rehabilitation, or due to improvements evident at the end of the program not being sustained to the outcome measurements points. The higher rate of influenza and pneumococcal vaccination in the intervention compared with the control group suggests improved delivery of care, but these differences were not statistically significant. Patients in the intervention group had higher COPD knowledge scores, presumably related to the education provided by the nurses, although the clinical importance of this is uncertain.

Our study had some limitations. The participating practices were drawn from a list of practices that had previous involvement in research or teaching, or that had attended continuing educational events. Therefore, they may not have been entirely representative of all practices in Australia. Sources of potential bias are that the uptake of the invitation to participate was $39.4 \%$, and that $26.8 \%$ of participants randomised were lost to follow-up at 12 months.

The criterion for entry into the study was having a diagnosis of COPD and we did not require this to be confirmed on spirometry. This reflects the real-world situation in primary care, where the diagnosis is often made and treatment initiated on clinical grounds. ${ }^{13-15}$ The rate of misclassification was similar to that of other studies from Australia ${ }^{16}$ and internationally. ${ }^{17,18}$

However, the intervention components were based on evidence of effectiveness from studies in patients with COPD confirmed on spirometry, and the intervention may only have been effective in this subgroup of participants. A subgroup analysis, which examined the outcomes for the 257 patients who had COPD confirmed on spirometry, was similar to the intention-to-treat analysis, with no statistically significant differences at 12 months in SGRQ, SF-12, lung function or smoking rates. As the numbers were smaller, there was a risk of a type 2 error, and this risk was further increased as the intracluster correlation found for the SGRQ (0.03) was slightly higher than our estimate of 0.01 . $^{18}$
Our findings are consistent with the current uncertainty about the effect of disease-management programs, including self-management support, for COPD. While some studies have shown benefit, ${ }^{6,19}$ others, including a recently published study of comprehensive care management to prevent COPD hospitalisations, have had negative results. ${ }^{20}$

The lack of impact from the intervention on prevalence of smoking demonstrates the need to continue to develop and test interventions to encourage smoking cessation in people with COPD. There is continuing debate about whether performing spirometry and informing patients of abnormal results increases smoking cessation. $^{21,22}$ There has been promising research on the use of lung age as a tool to encourage quitting, but this has not been studied in patients with COPD. ${ }^{23}$ The evidence base on smoking-cessation interventions for people with COPD is very limited, ${ }^{24}$ and there is a need for studies that evaluate both psychosocial approaches and innovative ways of using pharmacotherapy. ${ }^{25}$

Pulmonary rehabilitation has the potential to improve health-related quality of life, but even in the intervention group, less than a third of patients attended pulmonary rehabilitation. This was consistent with previous research reporting uptake of $33 \%-39 \%$ in pulmonary rehabilitation programs provided in outpatient clinics. $^{26}$ There is evidence that homebased programs may be as effective as supervised hospital outpatient-based programs, ${ }^{27,28}$ but studies on implementation are lacking. Finally, there is the question of whether the nurse-GP partnership intervention to implement evidence-based care would have been effective if it had been implemented with patients at an early stage 
of the disease. It has been suggested, for example, that the benefit of smoking cessation may be greatest in asymptomatic patients with measureable lung function impairment. ${ }^{29}$

The intervention tested in this study showed promise in that there were improvements in process of care, but it did not have a measurable impact on disease-related quality of life, respiratory function or smoking status. Given the burden that COPD places on individuals and society, and the importance of improved care in the community, further research to identify effective interventions, including examination of their costeffectiveness, is needed. This could involve more intensive interventions to support smoking cessation, new ways of delivering pulmonary rehabilitation, and intervention soon after diagnosis of COPD.

Acknowledgements: We acknowledge the contribution of labal Hasan to data collection, the nurses, GPs and patients who participated in the study, and the Divisions of General Practice who supported the study. Funding was from the National Health and Medical Research Council.

Competing interests: No relevant disclosures.

Received 17 May 2012, accepted 23 Aug 2012.

1 Price D, Duerden M. Chronic obstructive pulmonary disease. BMJ 2003; 326: 1046-1047.

2 Global Initiative for Chronic Obstructive Lung Disease (GOLD). Global strategy for the diagnosis, management and prevention of COPD. Updated Dec 2011. http://www.goldcopd.org (accessed Aug 2012).

3 McKenzie DK, Abramson M, Crockett AJ, et al; The Australian Lung Foundation. The COPD-X Plan: Australian and New Zealand guidelines for the management of chronic obstructive pulmonary disease 2011. http:www.copdx.org.au (accessed Aug 2012).

4 Taylor SJ, Candy B, Bryar RM, et al. Effectiveness of innovations in nurse led chronic disease management for patients with chronic obstructive pulmonary disease: systematic review of evidence. BMJ 2005; 331: 485

5 Wong CX, Carson KV, Smith BJ. Home care by outreach nursing for chronic obstructive pulmonary disease. Cochrane Database Syst Rev 2011; (3): CD000994.

6 Rea H, McAuley S, Stewart A, et al. A chronic disease management programme can reduce days in hospital for patients with chronic obstructive pulmonary disease. Intern Med J 2004; 34: 608-614

7 Hermiz O, Comino E, Marks G, et al. Randomised controlled trial of home based care of patients with chronic obstructive pulmonary disease. $B M$ 2002; 325: 938.

8 Zwar N, Hermiz O, Hasan I, et al. A cluster randomised controlled trial of nurse and GP partnership for care of chronic obstructive pulmonary disease. BMC Pulm Med 2008; 8: 8.

9 Jones PW, Quirk FH, Baveystock CM, Littlejohns P. A self-complete measure of health status for chronic airflow limitation. The St. George's Respiratory Questionnaire. Am Rev Respir Dis 1992; 145: 1321-1327.

10 Casaburi R, Mahler DA, Jones PW, et al. A longterm evaluation of once-daily tiotropium in chronic obstructive pulmonary disease. Eur Respir J 2002; 19: 217-224.

11 Zwar NA, Marks GB, Hermiz O, et al. Predictors of accuracy of diagnosis of chronic obstructive pulmonary disease in general practice. Med $J$ Aust 2011; 195: 168-171.

12 Lacasse Y, Goldstein R, Lasserson TJ, Martin S. Pulmonary rehabilitation for chronic obstructive pulmonary disease. Cochrane Database Syst Rev 2006; (4): CD003793.

13 Walters JA, Hansen E, Mudge P, et al. Barriers to use of spirometry in general practice. Aust Fam Physician 2005; 34: 201-203.

14 Poels PJP, Schermer TRJ, van Weel C, Calverley PMA. Spirometry in chronic obstructive pulmonary disease. BMJ 2006; 333: 870-871.

15 Walters J, Walters EH, Nelson M, et al. Factors associated with misdiagnosis of COPD in primary care. Prim Care Respir J 2011; 20: 396-402

16 Tinkelman DG, Price DB, Nordyke RJ, Halbert RJ. Misdiagnosis of COPD and asthma in primary care patients 40 years of age and over. J Asthma 2006; 43: 75-80.

17 Jones RC, Dickson-Spillmann M, Mather MJ, et al. Accuracy of diagnostic registers and management of chronic obstructive pulmonary disease: the Devon primary care audit. Respir Res 2008; 9: 62.

18 Kerry SM, Bland JM. Trials which randomize practices Il: sample size. Family Practice 1998; 15: 84-87.

19 Bourbeau J, Julien M, Maltais F, et al. Reduction of hospital utilization in patients with chronic obstructive pulmonary disease: a diseasespecific self-management intervention. Arch Intern Med 2003; 163: 585-591.

20 Fan VS, Gaziano M, Lew R, et al. A comprehensive care management program to prevent chronic obstructive pulmonary disease hospitalizations. Ann Int Med 2012; 156: 673-683.

21 Wilt TJ, Niewoehner D, Kane RL, et al. Spirometry as a motivational tool to improve smoking cessation rates: a systematic review of the literature. Nicotine Tob Res 2007: 9: 21-32.

22 Kotz D, Wesseling G, Huibers MJ, van Schayck OC. Efficacy of confronting smokers with airflow limitation for smoking cessation. Eur Respir J 2009; 33: 754-62.

23 Parkes G, Greenhalgh T, Griffin M, Dent R. Effect on smoking quit rate of telling patients their lung age: the Step2quit randomised controlled trial. BMJ 2008; 336: 598-600.

24 van der Meer RM, Wagena EJ, Ostelo RW, et al. Smoking cessation for chronic obstructive pulmonary disease. Cochrane Database Syst Rev 2003; (2): CD002999.

25 Moore D, Aveyard P, Connock M, et al. Effectiveness and safety of nicotine replacement therapy assisted reduction to stop smoking: systematic review and meta-analysis. BMJ 2009; 338: b1024.

26 Harris D, Hayter M, Allender S. Improving the uptake of pulmonary rehabilitation in patients with COPD: qualitative study of experiences and attitudes. Br J Gen Pract 2008; 58: 703-710.

27 Ashworth NL, Chad KE, Harrison EL, et al. Home versus center based physical activity programs in older adults. Cochrane Database Syst Rev 2005; (1): CD004017.

28 Maltais F, Bourbeau J, Shapiro S, et al. Effects of home-based pulmonary rehabilitation in patients with chronic obstructive pulmonary disease: a randomized trial. Ann Intern Med 2008 149: 869-878.

29 Price D, Freeman D, Cleland J, et al. Earlier diagnosis and earlier treatment of COPD in primary care. Prim Care Respir J 2011; 20: 15-22. 口

\section{What is a QR code?}

You may have noticed a strange symbol like the one here on some pages of the Journal (see pages 383 and 403) or in other places, such as on bus-stop ads or at the counter at cafes. These are called quick response (QR) codes, which work similarly to barcodes, in that they can digitally encode a range of information. They can be scanned by your tablet or smartphone and, in the Journal, they will take you directly to multimedia content such as podcasts without you having to type in a web address. The one on this page will take you to the MJA homepage.

\section{How do I scan QR codes?}

You need a tablet or smartphone and a QR reader to scan $Q R$ codes.

1) First download a free app that will scan $Q R$ codes. Go to the appropriate source of apps for your device (eg, Android Market, the App Store or Google Play) and download a free QR code reader such as i-nigma, RedLaser or Barcode Scanner.

2) Install the scanner app to your device.

3) With the scanner app open, hold your tablet or smartphone over the QR code.

\section{QR codes in the MJA}

You may see QR codes in the margins of MJA articles or in some advertising, which indicates that there is more information or multimedia content such as a podcast or video associated with that article. When you scan the QR code, your device will be taken to the website for that extra content, so that you can read, download or live stream the content. 
Flow diagram for the chronic obstructive pulmonary disease study, December 2006 - May 2009

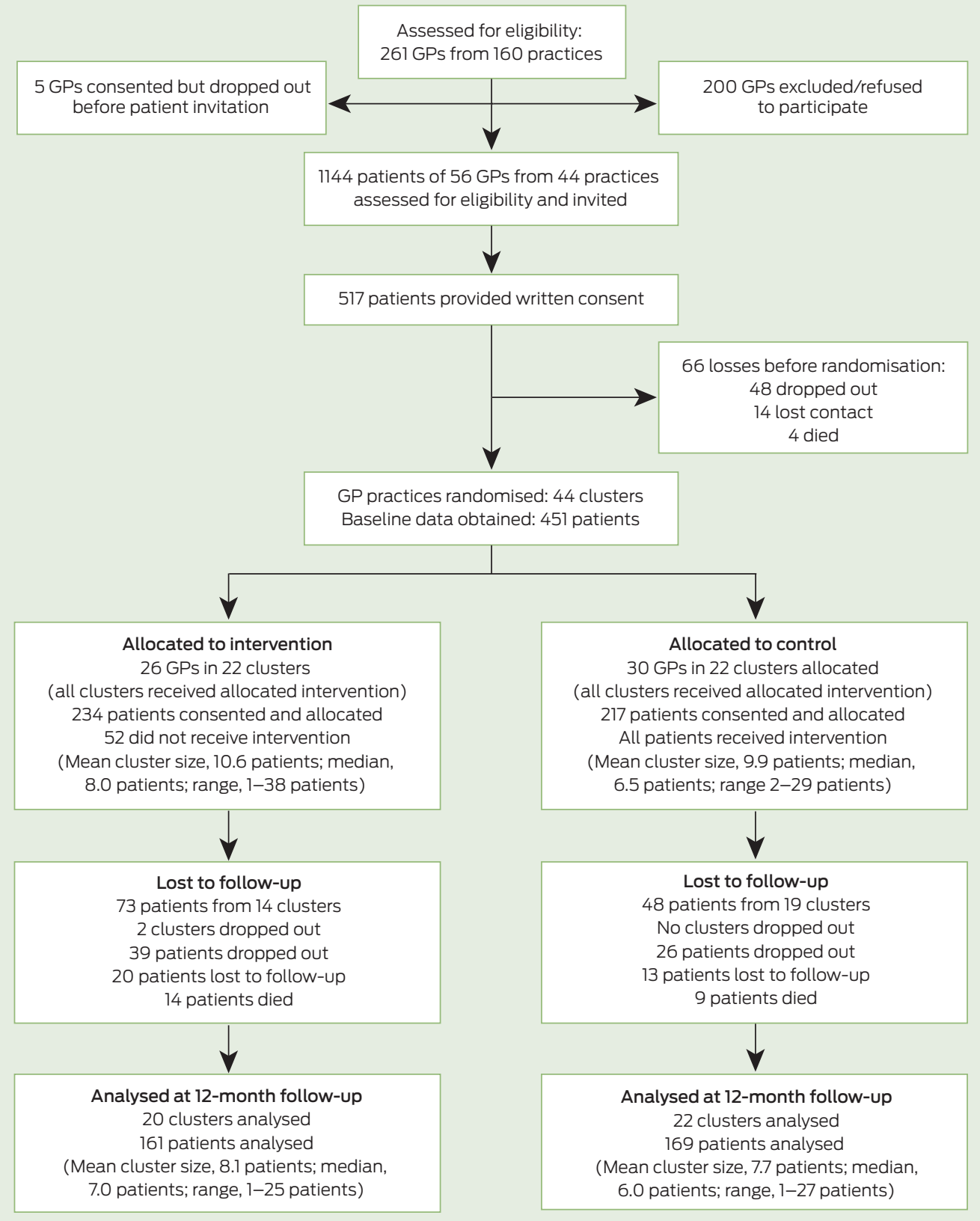




\section{Box unedited, as supplied by the authors}

\section{Intervention description}

Two intervention nurses with specific training worked in partnership with GPs, patients and other care providers over a six month period. Behaviour change was encouraged through use of motivational interviewing. Self management support was provided in the form of assistance with goal setting and action planning.

The intervention comprised

- An initial home visit involving comprehensive assessment, including pre and post bronchodilator spirometry.

- Development of a personalised care plan based on the recommendations of the COPDX guidelines using an electronic template provided. The care plan was based on the nurse assessment and discussion with the patient of goal setting and action planning. It the contained relevant components of smoking cessation, influenza and pneumococcal immunisation, pulmonary rehabilitation, medication review, nutrition, psychosocial issues, patient education, comorbidities and complications of COPD. Where spirometry did not confirm COPD the nurse discussed with the GP the actions following this and what parts of the care plan applied to these patients.

- The nurse worked with the patient, their GP and other health professionals to implement the plan. This involved at least two home visits and five telephone contacts from the nurse and a minimum of two consultations with their GP. The nurse facilitated referral and teamwork with other services as needed such as smoking cessation program, pulmonary rehabilitation program, pharmacist, specialist physician, Action Plans for exacerbations were discussed and patients were encouraged to take these to their GPs for completion.

- At the end of the six month intervention period progress against the goals in the plan were noted and a copy of the plan with these annotations provided to the GP. The completion of the plan was used to define that the patient had received the intervention. 